\title{
Whole transcriptome analysis of a reversible neurodegenerative process in Drosophila reveals potential neuroprotective genes
}

María José Ferreiro ${ }^{1}$, Naiara Rodríguez-Ezpeleta ${ }^{2,3}$, Coralia Pérez ${ }^{4}$, Michael Hackenberg ${ }^{5}$, Ana María Aransay², Rosa Barrio ${ }^{4^{*}}$ and Rafael Cantera ${ }^{1,6^{*}}$

\begin{abstract}
Background: Neurodegenerative diseases are progressive and irreversible and they can be initiated by mutations in specific genes. Spalt-like genes (Sall) encode transcription factors expressed in the central nervous system. In humans, SALL mutations are associated with hereditary syndromes characterized by mental retardation, sensorineural deafness and motoneuron problems, among others. Drosophila sall mutants exhibit severe neurodegeneration of the central nervous system at embryonic stage 16, which surprisingly reverts later in development at embryonic stage 17, suggesting a potential to recover from neurodegeneration. We hypothesize that this recovery is mediated by a reorganization of the transcriptome counteracting SALL lost. To identify genes associated to neurodegeneration and neuroprotection, we used mRNA-Seq to compare the transcriptome of Drosophila sall mutant and wild type embryos from neurodegeneration and reversal stages.
\end{abstract}

Results: Neurodegeneration stage is associated with transcriptional changes in 220 genes, of which only 5\% were already described as relevant for neurodegeneration. Genes related to the groups of Redox, Lifespan/Aging and Mitochondrial diseases are significantly represented at this stage. By contrast, neurodegeneration reversal stage is associated with significant changes in 480 genes, including 424 not previously associated with neuroprotection. Immune response and Salt stress are the most represented groups at this stage.

Conclusions: We identify new genes associated to neurodegeneration and neuroprotection by using an mRNA-Seq approach. The strong homology between Drosophila and human genes raises the possibility to unveil novel genes involved in neurodegeneration and neuroprotection also in humans.

Keywords: mRNA-Seq, Spalt, SALL, Neurodegeneration, Neuroprotection, Drosophila

\section{Background}

Neurodegenerative processes, which affect a high proportion of the human population worldwide, result from very complex interactions at molecular, cellular, histological and organismal levels, being progressive as well as irreversible. A great proportion of the human transcriptome is expressed in the central nervous system (CNS) under a strict spatial, temporal and quantitative control. Mutations in genes involved in a variety of molecular and cellular functions, resulting in abnormally

\footnotetext{
* Correspondence: rbarrio@cicbiogune.es; rcantera@zoologi.su.se

${ }^{4}$ Functional Genomics, CIC bioGUNE, Derio, Spain

'Developmental Neurobiology, IIBCE, Montevideo, Uruguay

Full list of author information is available at the end of the article
}

low or high levels of their corresponding proteins, contribute to the development of some important neurodegenerative diseases such as Alzheimer or Parkinson (reviewed in [1]).

The evolutionary conservation of key mechanisms for the development, function and maintenance of the nervous tissue makes the fly Drosophila melanogaster a good model system for the study of human neurodegenerative diseases [1-3]. The use of this model presents various additional advantages, including the increasing number of available genetic tools to manipulate gene expression, with temporal and spatial specificity, through trans-genes encoding wild type (WT) or mutated forms of Drosophila or human genes [4]. 
The zinc finger transcription factors Spalt-like (SALL) are expressed in the CNS during embryonic development in Drosophila and other organisms. In humans, mutations in SALL1 are associated with Townes Brocks Syndrome [5], while mutations in the SALL4 gene are associated with the Duane-Radial Ray or Okihiro Syndrome [6,7]. These syndromes are characterized by limb and renal malformations, as well as nervous system defects that result in sensorineural deafness and, in some cases, mental retardation and motoneuron problems $[6,8,9]$.

Two members of the SALL family are known in Drosophila, spalt major (salm) and spalt-related (salr) [10]. The transcription of salm starts shortly after blastoderm formation and continues throughout embryogenesis, overlapping partially with salr [11]. salm and salr null mutations affect several tissues, including the tracheal system [12], CNS [13] and the peripheral nervous system [14], and are embryonic lethal in homozygosis. Homozygous sall mutant embryos exhibit degeneration of the CNS at embryonic stage 16 (13-16 hours of development, Figure 1 A, B, D). In situ studies of sall mutant embryos and in vitro studies of neurons generated by sall mutant stem cells, including time-lapse video recording, demonstrated that the mutant phenotype exhibits fragility of the nervous tissue, deficient axonal cytoskeleton and loss of cell adhesion. Consistent with these findings, a study with transmission electron microscopy showed a greatly enlarged extracellular space and several other features indicative of a degenerative process, including vacuolization and abundant membrane "whorls" and autophagosomes [13]. This suggested that sall controls, directly or indirectly, the transcription of genes that are important for the integrity of the CNS, possibly acting through cell adhesion and the cytoskeleton. A few hours later, at early stage 17 (16-18 hours of embryonic development, Figure 1 A, C, E), part of this phenotype is reverted, suggesting a potential to recover from the neurodegenerative process. The recovery could be mediated by genetic redundancy, either as coincidental redundancy (i.e. genes performing the same function at the same developmental stage) or sequential redundancy (genes performing a similar function at different stages). In this last case, the disappearance of the ultrastructural sall phenotype in a few hours (from early stage 16 to early stage 17 ) could be partially explained by transcriptional changes taking place during this interval [13]. Genomic studies have subsequently reinforced this idea by showing that, during this time window, the Drosophila transcriptome undergoes rapid global changes in gene expression [15-17].

Based on the hypothesis that the recovery from neurodegeneration could be explained by a reorganization of the sall mutant transcriptome that partially compensates the absence of sall genes [13], we used mRNA high throughput sequencing (mRNA-Seq) to identify genes that might have neuroprotective function, by comparing the transcriptome of Drosophila sall mutant and wild type embryos from stages 16 and 17.

Our mRNA-Seq analysis demonstrates that a characteristic transcriptional signature identifies the neurodegeneration and reversal stages. Thus, the neurodegeneration phenotype observed at embryonic stage 16 in sall null mutants is associated with transcriptional changes in genes related to the Redox, Lifespan/ Aging and Mitochondrial diseases groups, while the reversal of the neurodegeneration observed at embryonic stage 17 is associated to the regulation of genes involved in the Immune and Salt stress response groups.

\section{Results}

\section{Analysis of the sall mutant transcriptome}

The mutation $D f(2 L) 32 F P-5$ used for the present approach is a small deficiency that covers both salm and salr genes [18]. Embryos that are homozygous mutant for these genes die at the end of embryogenesis or beginning of the first larval instar.

Total RNA was extracted from wild type (WT), $D f(2 L)$ $32 F P-5 /+$ (heterozygous, from now on called $\mathrm{He}$ ) or $D f$ (2 L) $32 F P-5 / D f(2 L) 32 F P-5$ (homozygous, from now on called $\mathrm{Ho}$ ) embryos at the two time points illustrated in Figure 1A. cDNAs from two or three independent poolpreparations for each genotype and developmental stage were sequenced. Sequencing parameters of the biological replicas of the studied genotypes are shown in Additional file 1: Table S1. DESeq package was used for measuring gene expression differences between the samples analyzed [19]. Genes showing expression differences among genotypes and developmental stages that exceeded the threshold defined as statistically significant $(p<0.001)$ were selected for further analysis. The variance in transcript representation between differential expression (DE) results per genotype comparison is shown in Additional file 2: Table S2. Alternatively, Reads Per Kilobase of transcript per Million mapped reads (RPKMs) were also calculated [20] to corroborate the expression variability intrasamples (Additional file 3: Table S3).

We first compared the global transcriptome profile of Ho sall mutant embryos with those of $\mathrm{He}$ and WT at embryonic stages 16 and 17.We hypothesized that transcriptional misregulation associated with neurodegeneration occurs mainly at stage 16 , and that genes providing neuroprotection will be regulated mainly at stage 17 . The heatmap in Figure 2A shows the hierarchical clustering of all genotypes at both stages with respect to the 2534 genes that showed differential expression in at least one of the compared pairs. These results show that the transcriptome differs greatly from stage 16 to 17 , as the 


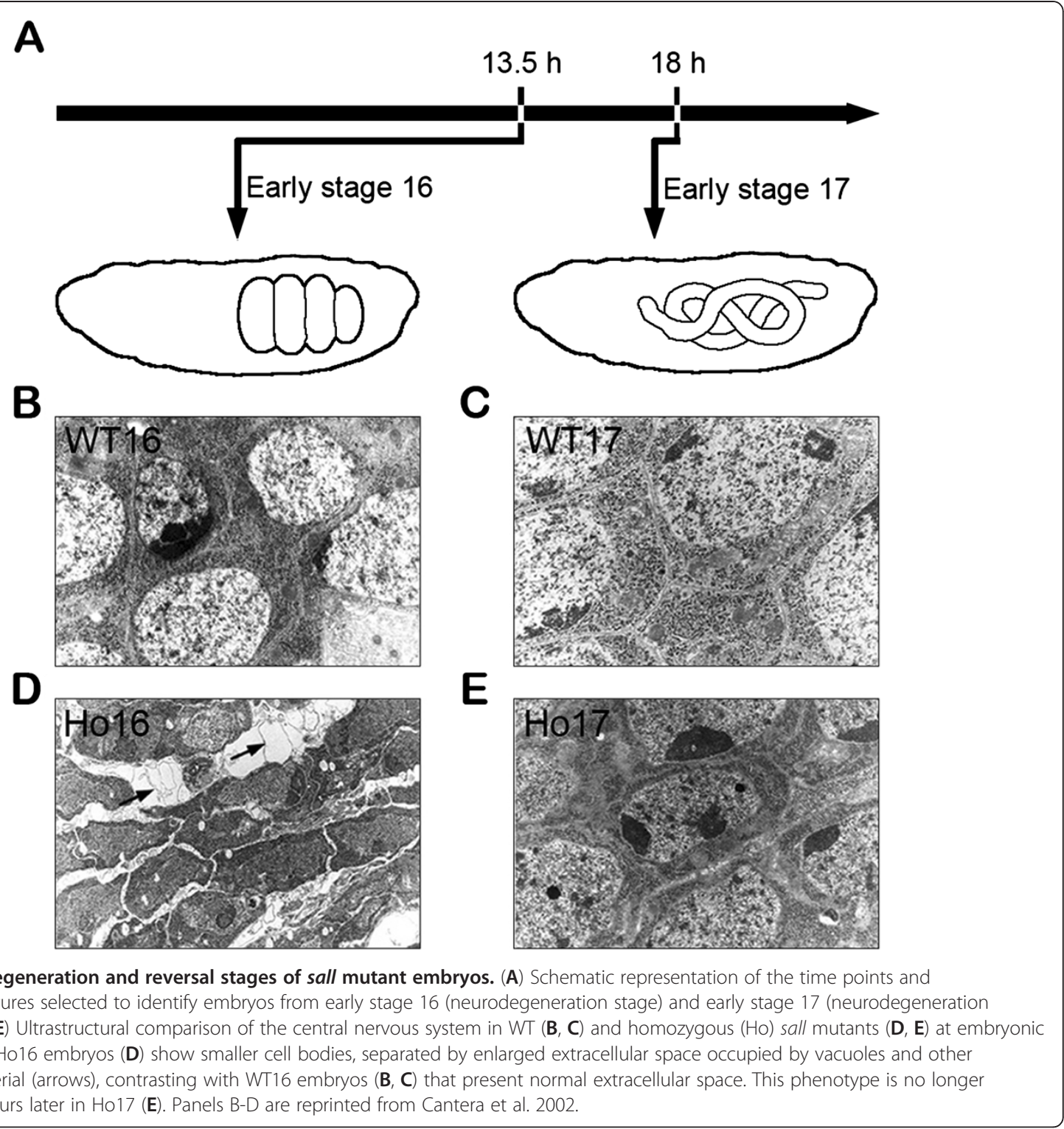

three genotypes at each stage segregate together and separate from the other stage.

The number of genes that showed differential expression profiles in each of the comparisons between WT16, Ho16, and He16 (Figure 2B) and WT17, Ho17, and He17 (Figure 2C) is indicated by Venn diagrams. The transcriptome profiles of $\mathrm{He}$ and WT embryos showed differences in less than 100 genes per stage (42 genes up and 30 down at stage 16; 24 genes up and 70 down at stage 17). The Ho mutants, instead, showed misregulation of more than 200 genes, most of which were upregulated (190 genes up and 26 down at stage 16; 163 genes up and 97 down at stage 17).

The transcriptome comparison of the three genotypes at stage 16 generated a list of 249 genes that were significantly misregulated in Ho16 or He16 sall mutants in comparison to WT16 (Figure 2B and Additional file 4: Table S4). Out of those, we discarded 161 genes with profiles not consistent among all genotype comparisons. For instance, genes that did not show significant differences between WT16 and Ho16, nor between He16 and Ho16, but showed significant differences between WT16 and He16 were removed from the analysis. The 88 remaining genes were classified in two categories: sall dose-independent (47 genes) and sall dose-dependent (41 genes), (Additional file 4).

The transcriptome comparison of the three genotypes at stage 17 generated a list of 307 genes significantly misregulated in Ho17 or He17 sall mutants with regard to WT17 (Figure 2C and Additional file 4). As mentioned previously for stage 16 , we discarded those genes with profiles not consistent among all genotypes comparisons (92 


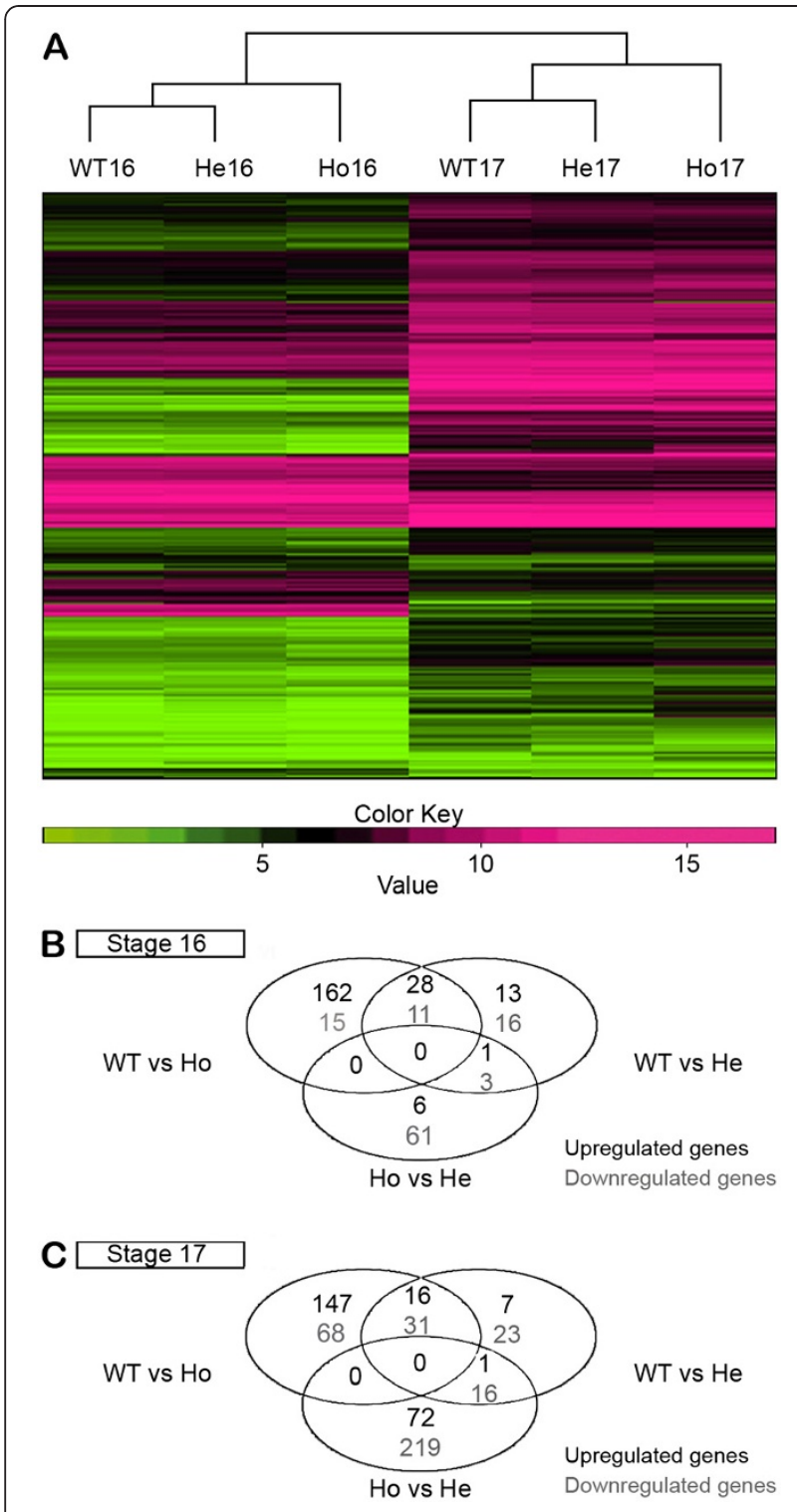

Figure 2 Comparative analysis of sall mutant transcriptome. (A) Heatmap representation of a filtered set of 2534 genes that show differential expression at $p<0.001$ in at least one of the following comparatives. At stages 16 or 17: WT vs homozygous (Ho), WT vs heterozygous ( $\mathrm{He}$ ) and $\mathrm{He}$ vs $\mathrm{Ho}$. At the transition from 16 to 17 : WT16 vs WT17, He16 vs He17, and Ho16 vs Ho17. Columns represent samples and rows genes. Colors represent log2 expression ratio values with pink being above and green below the row/column median level of expression (normalized gene counts) as shown by the scale (pink: genes upregulated; green: genes downregulated). (BC) Venn diagrams of differential expression overlap between WT, He and Ho sall mutant embryos at stage 16 (B) and 17 (C). Numbers represent upregulated and downregulated genes (top and bottom, respectively) in the second genotype with respect to the first (e.g. WT vs Ho represent the number of misregulated genes in homozygous compared to WT). genes). The remaining 215 genes were classified in two categories: sall dose-independent (151 genes) and sall dose-dependent (64 genes) (Additional file 4).

We found a dosage effect of Sall on certain genes, consistent with previous results [13]. He sall mutant embryos have higher transcript levels than Ho for armadillo, cadherin- $N$ and fasciclin- 2 (Additional file 5: Figure S1A). We also found intermediate transcript values for other genes in $\mathrm{He}$ embryos compared to those of Ho and WT (Additional file 5: Figure S1B-C). Among these, at stage 16, we observed genes associated with tissue regeneration (CG2233, [21]), Lifespan/aging (mtND2, [22]; dro5, [23]), and salt stress (sala, [24]). At stage 17 , some of the genes showing a dosage effect are associated with neurodegeneration and salt stress (IM10, $[24,25])$, or starvation (CG6283, [26,27]). Interestingly, in humans the Duane-Radial Ray Syndrome is caused by the deletion of one copy of SALL4, suggesting that also in humans two copies of a $S A L L$ gene are necessary to keep the correct levels of its downstream targets [28]. Furthermore, it is reported the dosage-dependent regulation of the target gene knirps by Sall in the Drosophila wing imaginal disc [29].

\section{Analysis of the transition between embryonic stages 16 and 17}

During the transition from early stage 16 to early stage 17 , the number of genes that change expression differed between WT and Ho sall mutant embryos (Figure 3). In WT embryos, this transition comprised the upregulation of 1687 genes and downregulation of 312 genes (Figure 3A). A similar change was detected in $\mathrm{He}$ sall mutant embryos (data not shown). However, Ho embryos showed less upregulated genes and more downregulated genes compared to WT (781 genes upregulated and 416 genes downregulated; Figure 3A). Despite these differences, Ho sall mutant embryos are able to pass to stage 17 (tubular intestine, Figure1A), and die shortly after. Therefore, the genes necessary for the transition from stages 16 to 17 are likely included in the 872 genes that change during the transition in the three genotypes (664 upregulated and 208 downregulated; Figure 3B).

\section{modENCODE comparison with differential expression results}

We compared our data with transcriptome data published by the modENCODE project [17]. We found good correlation (Pearson product-moment correlation coefficient) between our mRNA-Seq data and that of modENCODE, comparing our WT16 data with E12-14hs data $(r=0.79776$, Figure $4 \mathrm{~A})$ and our WT17 data with E18-20hs $(r=0.81202$, Figure 4B). 1175 genes are shared 

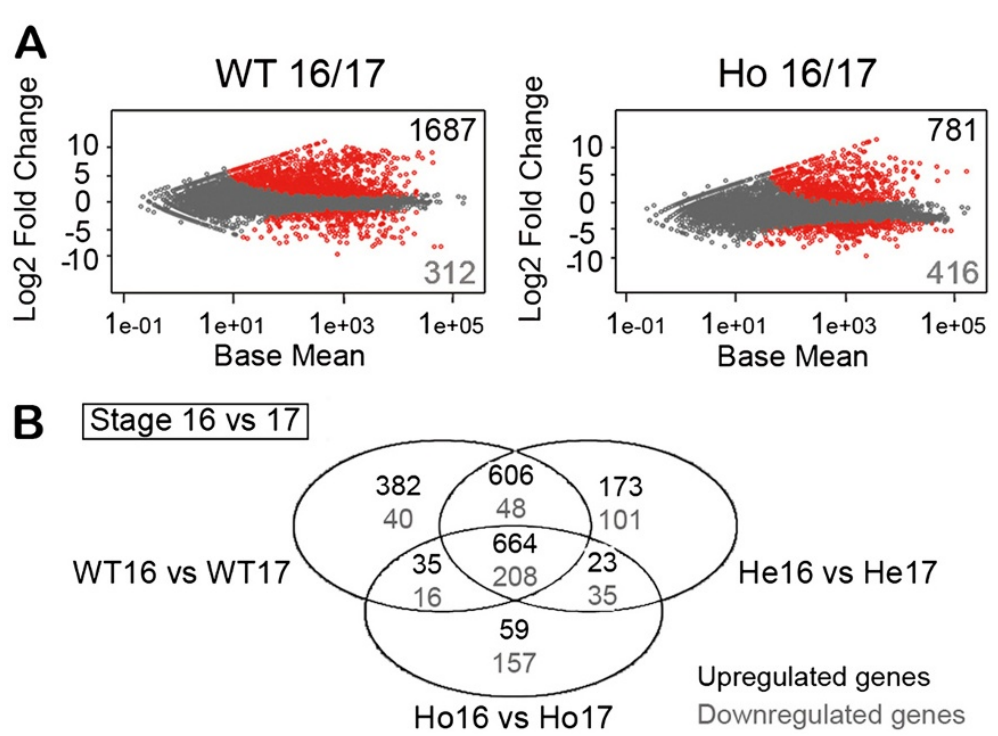

Figure 3 Differential expression analysis during the transition from embryonic stage 16 to 17 . (A) Analysis of the transcriptome of WT and Ho sall mutant embryos during the transition from stage 16 to 17. Scatter plot of log2 fold gene expression changes from the first to the second condition versus the mean counts for each compared pair. Unique counts normalized by the effective library size were used. Each point corresponds to a gene. Genes without significant change in their expression levels appear along the zero value of the $y$-axis (grey), and those whose transcription exceeds the threshold defined as statistically significant ( $p<0.001$, differentially expressed at $0.1 \%$ False Discovery Rate) are shown in red, above the $y$-axis zero value (genes upregulated at the transition from stage 16 to 17, number in black) or below (genes downregulated at this stage transition, number in grey). (B) Venn diagrams of differential expression overlap during the transition 16 to 17 of all genotypes analyzed. Genotypes analyzed: WT16 vs WT17, He16 vs He17, and Ho16 vs Ho17. Numbers indicate upregulated and downregulated genes for each comparison (top and bottom numbers, respectively) at stage 17 with respect to 16.

among the upregulated (64.35\%) and 412 genes among the downregulated (39.16\%) in both datasets.

\section{Validation of differential expression results}

In order to validate our results, we performed Q-PCR for selected genes that presented different number of reads per genotype and stage (Additional file 6: Figure $\mathrm{S} 2$ ). We chose two genes belonging to the Immune response group that showed different profiles in the mutant. According to our sequencing results, dro5 was highly upregulated in Ho16, but not in Ho17. These differences were confirmed by Q-PCR (Figure 4C). Conversely, according to our mRNA-Seq results, IM3 did not show significantly different expression levels between Ho16 and WT16, but was highly upregulated in the mutants at stage 17 . The same results were obtained by Q-PCR (Figure 4D). Interestingly, we noted that IM3 and dro5 genes show Sall binding sites in their promoters, and that those sites are conserved among several Drosophila species (Additional file 7: Figure S3).

\section{Identification of genes potentially involved in neurodegeneration}

The transcriptome comparison of WT and homozygous sall mutant embryos generated a list of 620 genes significantly misregulated at stages 16 and/or 17, including those that changed during the transition 16 to 17 exclusively in the homozygous embryos (Additional file 8: Table S5). The Additional file 9 shows the complete list of Gene Ontology (GO) categories enriched among these 620 genes, after a non-biased analysis with VLAD online tool (http://proto.informatics.jax.org/prototypes/ vlad-1.0.3). This analysis revealed an enrichment of genes involved in the oxidation-reduction process in the WT16 vs Ho16 comparison, as well as an enrichment of serine-type endopeptidases in the WT17 vs Ho17 and the Ho16 vs Ho17 comparisons. Next, we performed a biased GO analysis of these 620 genes, using functional categories selected after an extensive literature search (Additional file 10: Tables S6, S8 and S10). Through our bibliographic search we were able to identify the involvement of some of these genes in neurodegeneration or neuroprotection, either in Drosophila or in other organisms (72 genes highlighted in red in Additional file 8). Our hypothesis assumes that changes in transcript levels in Ho16 in comparison to WT16 (Figure 2B) might reveal genes contributing directly or indirectly to the neurodegenerative phenotype as well, as other genes with several other functions not relevant for this study.

Classification of all the genes misregulated in Ho16 with respect to WT16 in Functional Groups revealed that $8 \%$ belong to the Neuronal group, $7 \%$ to the Redox group, followed by Salt stress (6\%), Tissue regeneration (6\%), Lifespan/Aging (6\%), and the Neurodegeneration/ 

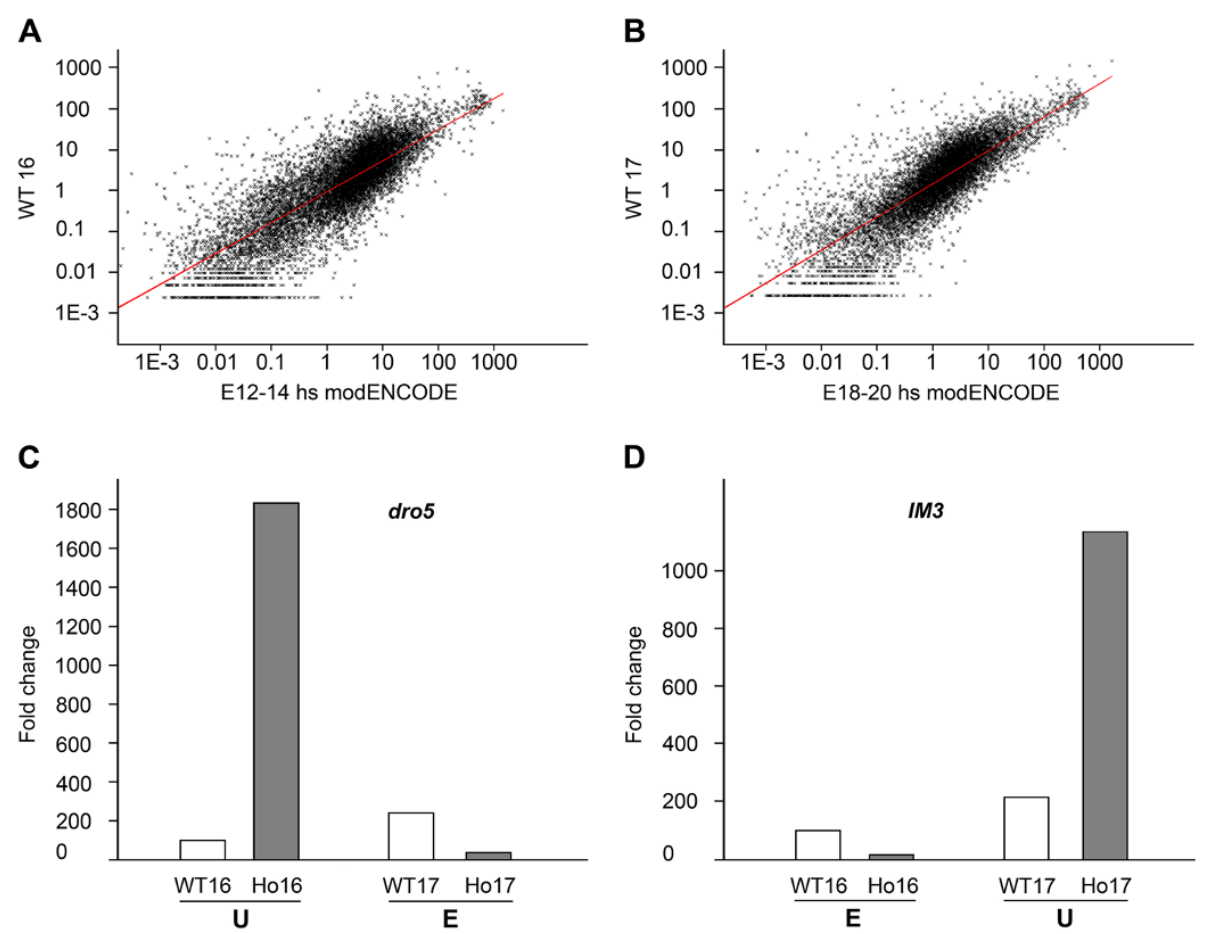

Figure 4 Validation of data from mRNA-Seq. (A, B) Graphical representation of the correlation between modENCODE expression data of E12$14 \mathrm{hs}$ embryos and the mRNA-Seq expression data for WT16 (A) and E18-20hs embryos with the mRNA-Seq expression data for WT17 (B). Correlation coefficients are 0.79776 and 0.81202 , respectively. (C, D) Graphical representation of the expression of dro5 (C) and IM3 (D) obtained by Q-PCR in the indicated genotypes. Ho: homozygous sall mutant embryos. U (upregulated) and $\mathrm{E}$ (equal) refer to the expression analysis by mRNA-Seq of these genes at the indicated genotypes' comparisons.

Neuroprotection groups (5\%), (Figure 5A and Additional file 10: Table S6), and by other groups with less than $5 \%$ representation. Gene Ontology (GO) analysis of these genes showed that the groups associated with Neurodegeneration/Neuroprotection, Redox, Lifespan/ Aging and Mitochondrial diseases were significantly represented $(\mathrm{p}<0.01)$ among Ho16 misregulated genes, with respect to their representation in the whole Drosophila genome (Figure 5A, highlighted in red). Neuronal and Starvation groups were also significantly represented $(\mathrm{p}<0.05$; Figure $5 \mathrm{~A}$, highlighted in green). Interestingly, $27 \%$ of genes misregulated in Ho16 embryos with respect to WT16 have no biological function experimentally assigned or suggested yet, and could be considered as new genes potentially associated to neurodegeneration.

Classification of genes that change transcript levels in Ho16 when compared to He16 in Functional Groups revealed that $8 \%$ belong to the Salt stress group, $7.5 \%$ to the Neuronal group, followed by Tissue regeneration (6\%), Mitochondrial diseases (6\%), Lifespan/Aging (6\%), and Redox (5\%), and by other groups with less than 5\% representation (Additional file 10: Table S7 and Additional file 11: Figure S4A). GO analysis of these genes showed that the Redox group was significantly represented $(\mathrm{p}<0.01)$ among Ho16 misregulated genes, with respect to their representation in the whole Drosophila genome (Additional file 11: Figure S4A, highlighted in red). Neuronal, Mitochondrial diseases, Neurodegeneration/Neuroprotection, Hormonal regulation and Starvation groups were also significantly represented ( $\mathrm{p}<0.05$; Additional file 11: Figure S4A, highlighted in green). These results are coincident with those showed in the WT16 vs Ho16 comparative (Figure 5A).

\section{Identification of genes potentially involved in neuroprotection}

Our hypothesis also assumes that among the genes with significantly altered transcript levels in Ho17 in comparison to WT17 (Figure 2C) might be those with neuroprotective function.

Classification of all these genes in Functional Groups showed that $10 \%$ of them belong to the Immune response group, $9 \%$ to the Neuronal group, followed by Salt stress (8.5\%), Neurodegeneration/Neuroprotection (6\%), Lifespan/Aging (5.5\%), and other functional groups (each representing less than $5 \%$ of the misregulated genes), (Figure 5B and Additional file 10: Table S8). GO analysis of these genes showed that Immune response, 


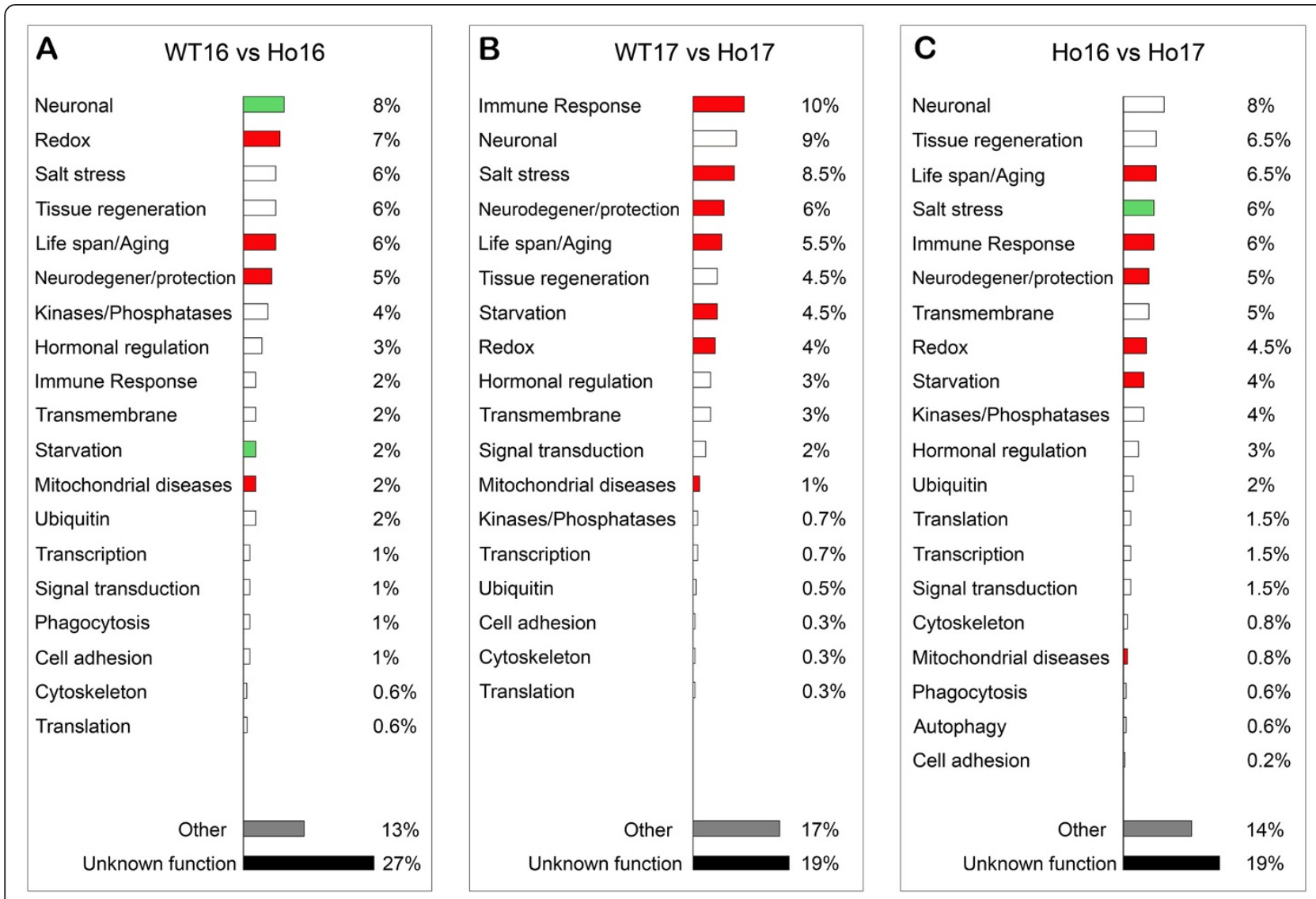

Figure $\mathbf{5}$ Classification of genes misregulated in homozygous sall mutant embryos in Functional Categories. (A-C) Graphic representation of the main functional groups enriched in misregulated genes in homozygous sall mutants (Ho), expressed as percentage of genes in each group. In red are marked the groups significantly overrepresented with respect to the total Drosophila genome with $p<0.01$ and in green with $p<0.05$. (A) Classification of the genes misregulated in Ho16 compared with WT16. (B) Classification of the genes misregulated in Ho17 compared to WT17. (C) Classification of the genes misregulated in Ho embryos at the transition from stage 16 to 17.

Salt stress, Neurodegeneration/Neuroprotection, Lifespan/ Aging, Starvation, Redox and Mitochondrial diseases groups were significantly overrepresented $(\mathrm{p}<0.01)$ among Ho17 misregulated genes, with respect to their representation in the whole Drosophila genome (Figure 5B, highlighted in red). The Neuronal group was also significantly overrepresented $(\mathrm{p}<0.05$; Figure $5 \mathrm{~B}$, highlighted in green). Interestingly, $19 \%$ of genes misregulated in Hol7 with respect to WT17 have no biological function experimentally assigned or suggested yet, and could be potentially associated to neuroprotection.

We also compared gene transcript levels in Ho17 with respect to He17 (Additional file 10: Table S9 and Additional file 11: Figure S4B). Classification of these genes in Functional Groups revealed that $10 \%$ belong to Salt stress and $10 \%$ to Immune response, followed by Mitochondrial diseases (7\%), Neuronal (6\%) and Lifespan/Aging (5\%), and by other groups with less than $5 \%$ representation. GO analysis of these genes showed that Salt stress, Immune response, Mitochondrial diseases, Neurodegeneration/ Neuroprotection and Starvation groups were significantly represented $(\mathrm{p}<0.01)$ among Ho17 misregulated genes, with respect to their representation in the whole Drosophila genome (Additional file 11: Figure S4B, highlighted in red). These results are coincident with those showed in Figure 5B. The homozygous embryos show overrepresentation of genes belonging to the same groups than the comparison between WT17 vs Ho17, supporting the concept that heterozygous embryos are more closely related to WT than to homozygotes.

Finally, our hypothesis assumes that genes with neuroprotective function might also be found among the genes with significantly altered transcript levels at the transition from stage 16 to 17 only in homozygous sall mutant embryos but not in WT or heterozygous (Figure 3B). Classification of all these genes in Functional Groups showed that $8 \%$ belong to the Neuronal group, $6.5 \%$ to Tissue regeneration, $6.5 \%$ to Lifespan/ Aging, $6 \%$ to Salt stress, $6 \%$ to Immune response, $5 \%$ to Neurodegeneration/Neuroprotection and 5\% to Transmembrane groups, followed by other groups with less than 5\% representation (Figure 5C and Additional file 
10: Table S10). GO analysis of these genes showed that the groups associated with Lifespan/Aging, Immune response, Neurodegeneration/Neuroprotection, Redox, Starvation and Mitochondrial diseases were significantly overrepresented $(\mathrm{p}<0.01)$ with respect to their incidence in the whole Drosophila genome (Figure 5C, highlighted in red). The Salt stress group was also significantly overrepresented $(\mathrm{p}<0.05$; Figure $5 \mathrm{C}$, highlighted in green). The Neurodegeneration/Neuroprotection and Redox groups were significantly overrepresented among downregulated genes $\left(\mathrm{p}=2.15 \times 10^{-09}\right.$ and $\mathrm{p}=1.29 \times 10^{-04}$, respectively), while the groups for Immune response, Starvation and Mitochondrial diseases were significantly overrepresented among upregulated genes $\left(\mathrm{p}=8.77 \times 10^{-12}, \mathrm{p}=2.75 \times 10^{-12}\right.$ and $\mathrm{p}=1.36 \times 10^{-05}$, respectively). Finally, $19 \%$ of genes misregulated in homozygous embryos at the transition between stages 16 and 17 have no biological function experimentally assigned or suggested yet, and could be considered as new genes potentially associated to neuroprotection.

When we analyzed the transcriptional changes in the 16 to 17 transition in WT embryos, we saw that only the Immune response, Starvation, Tissue regeneration and Mitochondrial diseases groups are overrepresented with respect to the whole genome (Additional file 10: Table S11 and Additional file 11: Figure S4C). However, this overrepresentation is in a lower proportion than in Ho16 vs Ho17 comparison, which is in agreement with our hypothesis that the transcriptional changes that we see in the 16 to 17 transition in homozygous embryos are specifically developed by the homozygous mutant embryos to try to compensate their neurodegeneration at stage 16.

\section{Discussion}

Analysis of the transition between embryonic stages 16 and 17

Several waves of coordinated down- or upregulation in the expression of large gene clusters have been detected throughout the life cycle of Drosophila using microarrays technology $[15,16,30]$. A major transcriptional shift occurs between 11 and 18 hours of embryonic development [16], which partially overlaps with the time interval studied here. Our study confirms that the Drosophila transcriptome changes quickly at the transition between stage 16 and 17 of embryonic development, within less than 45 hours, and shows that a major shift occurs also in sall heterozygous and homozygous mutants, although there are clear differences in the transcriptome of each genotype. The 872 genes that we found to change transcript levels from stage 16 to 17 in all three genotypes studied here, most probably include all or most of the genes that are truly necessary for this developmental transition.

\section{Analysis of the sall mutant transcriptome}

The transcriptome of homozygous sall mutant embryos was clearly different from those of heterozygous and WT embryos at both stages. Most of the genes misregulated in homozygous mutants were upregulated at both stages, in agreement with studies showing that Sall proteins act as transcriptional repressors in Drosophila and vertebrates [31-33]. The finding that the majority of misregulated genes in embryos with a neurodegenerative phenotype was upregulated adheres to a general trend established by genomic studies of neurodegenerative processes in Drosophila CNS tissue, for example, in parkin mutants [34] and in flies with neurodegeneration caused by transgenic expression of alpha-synuclein or mutated tau [25]. The opposite relationship was found in flies with retinal degeneration caused by polyglutamine expression [35].

\section{Identification of genes potentially involved in neurodegeneration}

In a previous study we described in detail the phenotype of sall mutant embryos, which includes several landmarks of degeneration, such as fragility of the nervous tissue, deficient axonal cytoskeleton, loss of cell adhesion, enlarged extracellular space, vacuolization and abundant membrane "whorls" and autophagosomes [13]. Other embryonic phenotypes have been described, including malformation of the tracheal [12] or the peripheral nervous system [14]. Even if some aspects of the phenotype could be attributed to reasons different than neurodegeneration, the prominent phenotype shown in the CNS prompted us to assume that genes significantly up or down-regulated at stages 16 and/or 17, and during the transition 16 to 17 specifically in the homozygous sall mutant embryos might include many of the genes associated with the neurodegenerative phenotype and its reversal.

Classification of all the genes whose transcription was misregulated in Ho16 with respect to WT16 in Functional Groups, revealed that only $5 \%$ of them have been previously associated to neurodegeneration. The overrepresentation of the Neurodegeneration and the Redox groups among Ho16 misregulated genes $\left(\mathrm{p}=1.28 \times 10^{-07}\right.$ and $\mathrm{p}=7.59 \times 10^{-07}$, respectively) is in accordance with previous works about neurodegeneration. The misregulated genes belonging to the Redox group include sugarless $(s g l)$, associated with the Wingless pathway and to resistance to oxidative damage [36,37]; l(2)01289, which protects from beta-amyloid toxicity [38]; nine genes codifying for cytochromes (mt: Cyt-b, mt: CoI-III, Cyp4g1, Cyp4p2, Cyp6a23, Cyp12c1, Cyp304a1) and six genes codifying for NADH-ubiquinone oxidoreductase chains (mt:ND1ND6). A predominance of the Redox group was also found in flies with neurodegeneration caused by mutation in parkin [34], expression of human beta-amyloid [38] or 
hyperoxia [39]. The Mitochondrial disease group was also strongly represented among Ho16 downregulated genes $\left(\mathrm{p}=5.25 \times 10^{-03}\right)$ and included among others technical knockout, a model for mitochondrial disease [40]. As a whole, these results reinforced the abundant data supporting the existence of a strong correlation between redox imbalance and neurodegeneration [41-45] and strongly indicate that SALL proteins are important for normal mitochondrial function. Interestingly, genes encoding mitochondrial proteins represent the major group of genes that are misregulated both, in our model, and in muscle tissue where salm expression was silenced [46] (Additional file 12: Table S12).

Gene Ontology analysis of all the genes misregulated in Ho16 with respect to WT16 showed that the groups Neuronal (genes associated with nervous system development and function) and Starvation were also significantly represented $(\mathrm{p}<0.005)$. Some examples are Ama, which encodes a protein important for neuronal cell adhesion (ligand of Neurotactin) also misregulated by polyglutamine expression [35]; Hsp23 and Hsp26, that encode heat shock proteins expressed in CNS and other tissues, previously associated with longevity [47], mitochondrial diseases [40] and tissue regeneration [21]; nicotinic Acetylcholine Receptor a 34E (nAcR $\alpha-34 E)$, that is expressed in the CNS of Drosophila larva and adult, encodes a protein with nicotinic acetylcholine-activated cation-selective channel activity; and CG4306, identified as a Drosophila Orb2 target gene, involved in neuronal growth, synapse formation, and protein turnover [48].

\section{Identification of genes potentially involved in neuroprotection}

The classification in Functional Groups of all the genes misregulated in Ho17 with respect to WT17 and in Ho17 with respect to Ho16, allowed us to identify genes potentially involved in the reversal of neurodegeneration. We found that $5 \%$ of these genes have already been associated with Neurodegeneration/Neuroprotection. $H s p 70 B c$ is a paradigmatic example, with ample documentation of a neuroprotective function in flies with neurodegenerative phenotypes caused by parkin mutations or other reasons (reviewed by $[39,49,50]$. We propose that many of the remaining Ho17 misregulated genes (95\%) are probably also relevant for neuroprotection.

The Immune group was particularly overrepresented among the genes upregulated in $\operatorname{Hol}\left(\mathrm{p}=6.48 \times 10^{-21}\right)$. Among others, we found upregulation of Ts $f 1$ and seven IM genes (Immune Induced Molecules: IM1-4, IM10, IM14, IM23) and downregulation of Ect3. Several of these genes (IM1, IM4, Tsf1) are regulated during Drosophila tissue regeneration [21]. We noticed that during normal development many of these genes show a sharp, very large peak of expression during metamorphosis [17] a time when the nervous system is radically remodeled. The importance of the Immune response group was previously demonstrated in other genomic studies of Drosophila neurodegeneration [25,34,38,39]. IM4 is the second most upregulated gene in parkin mutants [34], IM10 is upregulated in flies with neurodegeneration caused by mutated human tau [25], and Ect3 is the homolog of GLB1, a human gene associated with mental retardation [51]. Other misregulated genes belonging to the Immune response group are several members of the Jonah gene family (Jon25Bi-iii, Jon44E, Jon65Ai and Jon99F), which encode proteases and have a clear involvement in proteolysis. Two of them (Jon25Bi and Jon25Bii) are also associated with mitochondrial function [40].

It has been suggested that the upregulation of immune genes in Drosophila parkin mutants might be related with inflammation and other immunological reactions seen in the nervous tissue of patients with neurodegenerative diseases. However, a note of caution is warranted. Individual genes can have several functions and those annotated within the immune group in Drosophila are not an exception. The kappaB transcription factors encoded by dorsal, dif and relish, for example, besides being regulators of the immune response to pathogens, are also key players in pathways that govern the development of the body axis, as well as the development and differentiation of blood, muscle and neuronal cells $[52,53]$. We believe that beyond the possible engagement of immune genes in broad stress responses, including those constitutive to neurodegenerative processes, some might have neuroprotective functions as suggested here by their upregulation during the reversal of the phenotype. Future studies should address these possibilities, taking advantage of the abundance of mutants and genetic tools available for this purpose in Drosophila.

In addition to the Immune response group, the Neurodegeneration/Neuroprotection group is also highly represented among Ho17 misregulated genes $\left(\mathrm{p}=1.05 \times 10^{-12}\right)$. Besides Hsp70Bc, named above, other examples of this group are Cyp6a8, CG2065, CG11825, GstE1, Prx2540-1 and Prx2540-2 that are associated to common neurodegenerative diseases, such as Parkinson and Alzheimer [25,34,38,39,54]. CG16727, Prx2540-1 and $\operatorname{Pr} \times 2540-2$ are additionally associated with the Mitochondrial diseases group [40], that is also well represented among Ho17 misregulated genes $(p=0.00165)$. We found some genes implicated in mental retardation, like $L K R$ [51], also implicated in the stress response to starvation (Starvation group). This last group is highly represented as well among Ho17 misregulated genes $\left(\mathrm{p}=8.36 \times 10^{-15}\right)$, by genes like Osi6, CG10814, GRHR, ACC, CG18135, CG10918, CG9757, Prx2540-1 and Prx2540-2 [26,55-57]. Edg91, GstE1 and caz are examples of genes misregulated in Ho17, also altered during 
tissue regeneration [21], while Cyp6a13, Nplp4, mthl8, Prx2540-1 and Prx2540-2 are other examples of Ho17 misregulated genes that have been associated with Lifespan/Aging group $\left(\mathrm{p}=1.96 \times 10^{-6} ;\right.$ [58-61] $)$.

\section{A salt-stress response might be activated during the reversal of neurodegeneration}

An interesting observation is that a substantial proportion (between 6 and 8.5\%) of the genes misregulated in Ho16 and Ho17 or during the transition between both stages was also misregulated in response to osmotic stress caused in WT flies by dietary administration of excessive salt [24]. This proportion is statistically significant with respect to the whole Drosophila genome among Ho17 upregulated genes $\left(\mathrm{p}=1 \times 10^{-8}\right)$. The abundant representation of genes upregulated during the response to salt stress [23] in our mutant samples and, in particular, their enrichment in homozygous embryos by stage 17, suggests that a salt-stress response might be activated during the reversal of the neurodegenerative phenotype. This correlation could explain the phenotype observed by electron microscopy in the brain of Ho16 embryos ([13]; Figure 1), because the extraordinary enlargement of the extracellular space, shrunken neuronal cell bodies and smaller axonal diameter observed at this stage, could represent an osmotic imbalance driving water from the cells. Later on, at stage 17 (neurodegeneration reversal), the upregulation of genes from the salt stress response could be part of the mechanism mediating the disappearance of this phenotype. If this hypothesis is correct, SALL proteins are dispensable for mounting a rapid response to a hyperosmotic condition, despite the fact that salm was one of the genes with highest upregulation 4 hours after the response to salt stress was initiated [24]. The correlation between salm and osmotic regulation is nevertheless intriguing, as mutations in human and mouse $S A L L$ genes have been firmly associated with severe pathologies of kidney development and function (reviewed in [10]).

\section{Conclusions}

The main contribution of this work is the identification of a set of genes with abnormally high or low number of transcripts during the reversal of a neurodegenerative phenotype (Figure 6), most of which could be newly associated either to neurodegeneration or neuroprotection. To our knowledge, this is the first study that employs mRNA-Seq to approach this issue, comparing the transcriptome of Drosophila embryonic CNS with a temporal resolution of less than 5 hours (stages 16 and 17 of embryonic development).

Our analysis included not only the transcriptomic comparison of homozygous sall mutant and WT embryos at neurodegeneration and reversal stages, but also with heterozygous sall mutants, which do not develop the neurodegenerative phenotype observed in the homozygous. This allowed us to better identified genes that could contribute to neuroprotection.

Interestingly, several of the identified genes at stages corresponding to neurodegeneration and its reversal are not yet functionally annotated and could be experimentally associated to neurodegeneration and/or neuroprotection in future studies. Our analysis also suggests for the first time, an involvement of Drosophila sall genes in maintaining or regulating normal mitochondrial function. In addition, our results suggest that a salt-stress response might be activated during the reversal of the neurodegenerative phenotype. This could be a general phenomenon mediating neuroprotection not only in our model of study, but also in other neurodegeneration processes, that should be addressed in further experiments.

Given the strong homology exhibited by Drosophila and human genes, our study might help to better understand human neurodegenerative diseases by unveiling novel genes involved in both neurodegeneration and neuroprotection.

\section{Methods}

\section{Selection of embryos and RNA extraction}

$D f(2 L) 32 F P-5$ corresponds to a small deficiency on chromosome 2 that lacks both salm and salr genes [31]. As $D f(2 L) 32 F P-5$ stock is homozygous lethal in late embryonic stages, this deficiency is maintained as an heterozygote stock with a $C y O-G F P$ balancer $(D f(2 L)$ $32 F P-5 / C y O-G F P)$, which also facilitates embryo selection by GFP expression. The mutant stock was backcrossed with the balancer stock until all the chromosomes, except the one carrying the mutation, were substituted before the selection of the embryos.

Embryos were collected at early stage 16 (13-14 hours of egg development at $25^{\circ} \mathrm{C}$ ) and early stage 17 (around 16 hours) based on midgut morphology and GFP expression (Figure 1A). WT embryos were selected from an Oregon $R$ fly stock. Homozygous $D f(2 L) 32 F P-5 / D f(2 L)$ $32 F P-5$ embryos were selected from a $D f(2 L) 32 F P-5 /$ CyO-GFP fly stock by the absence of GFP. Heterozygous $D f(2 L) 32 F P-5 /+$ embryos were also selected by the absence of GFP from the F1 progeny of $D f(2 L)$ 32FP-5/CyO-GFP females crossed with white- males. Fifty embryos per tube were collected in $20 \mu \mathrm{l}$ of RNAlater (Ambion) and frozen in liquid nitrogen. Between two and three different pools (50 embryos each) were chosen per genotype and stage to obtain biological replicates for each condition.

Total RNA extracts were obtained from all samples, using the "Cells to cDNA II" kit (Ambion) according to the manufacturer's instructions, quantified using Nanodrop (Thermo Scientific) and quality assessed by Agilent 2100 Bioanalyzer (Agilent). 


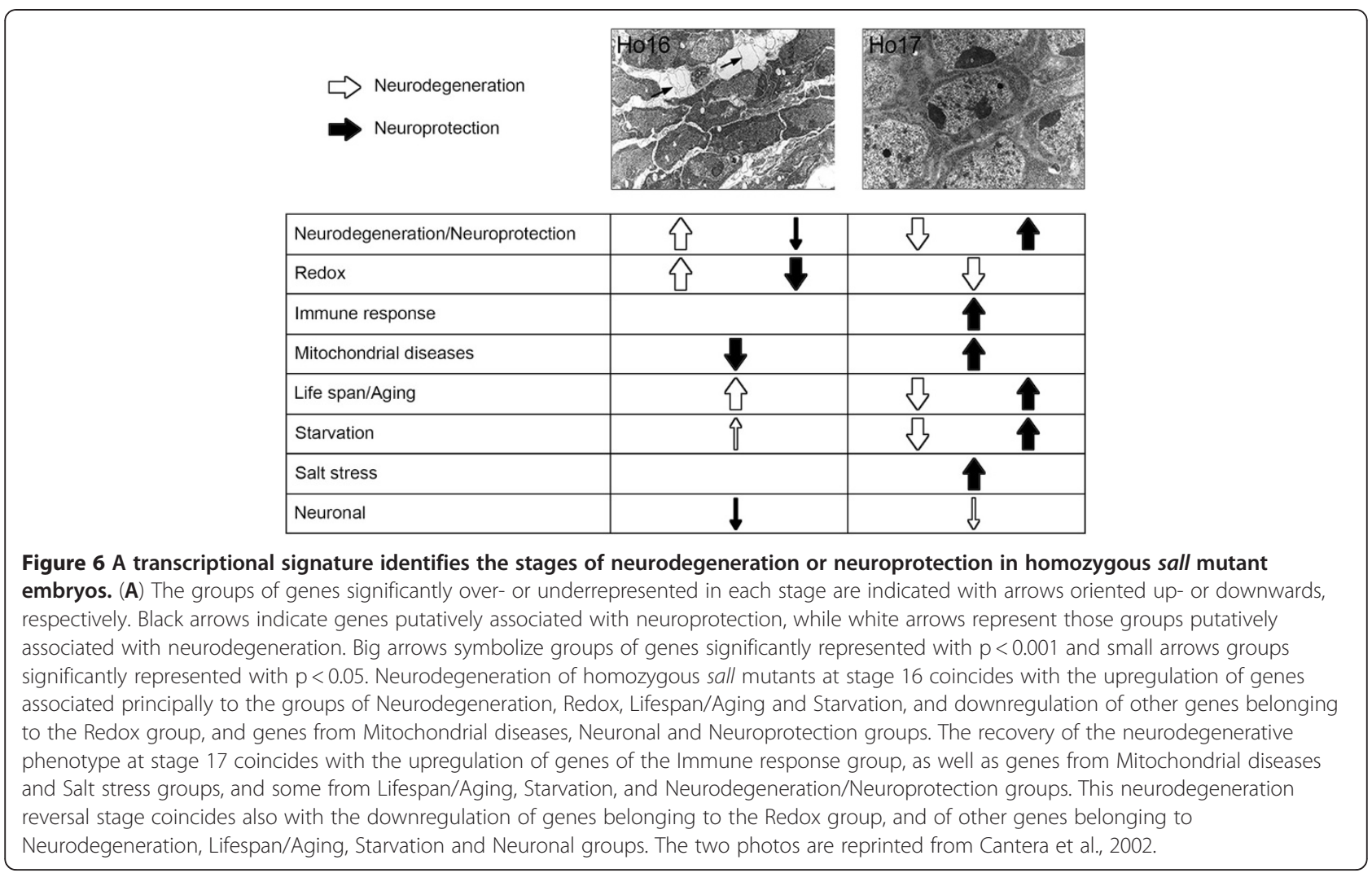

\section{Expression analysis by PCR}

Mutant samples were confirmed by PCR analysis of sall expression. Selected genes were validated by Q-PCR in independent RNA samples per genotype. RNA samples were first treated with $2 \mu \mathrm{l}$ of DNaseI for 15 minutes at $37^{\circ} \mathrm{C}$ followed by enzyme inactivation for 5 minutes at $75^{\circ} \mathrm{C}$. Two micrograms of RNA were retrotranscribed with random primers using the SuperScript III FirstStrand Synthesis System (Invitrogen) at a $100 \mu$ l volume per reaction, following manufacturer's instructions. Specific oligonucleotides for salm, dro5, IM3 and RpL32 were designed using the primer blast tool from NCBI website [62] (Additional file 13: Table S13). RpL32 was used as a control.

For Q-PCR, EvaGreen master mix (Biotium) and miHot Taq mix (Metabion) were used. Reactions were performed in $20 \mu$ l reaction volume (20 ng of cDNA, 1x mi-Hot Taq mix, 1x Eva Green mix, $0.1 \mu \mathrm{M}$ of each primer Forward and Reverse) in a CFX-thermocycler (BioRad) as follows: $95^{\circ} \mathrm{C}$ for $10 \mathrm{~min}, 40$ cycles of $95^{\circ} \mathrm{C}$ for 15 seconds and $60^{\circ} \mathrm{C}$ for $1 \mathrm{~min}$, and a final extension of $95^{\circ} \mathrm{C}$ for $1 \mathrm{~min}$. A melt curve from 60 to $95^{\circ} \mathrm{C}$, with $0.5^{\circ} \mathrm{C}$ temperature increment every 5 seconds, was carried out. All the reactions were run in agarose gels stained with ethidium bromide for validation of amplification specificity. RpL32 was used as a control.
RNA sequencing, annotation and differential expression cDNAs from two or three independent poolpreparations per genotype and developmental stage were sequenced. mRNA-Seq libraries were obtained out of $1 \mu \mathrm{g}$ of total RNA per pool following the mRNA Sequencing Sample Preparation kit's instructions (Illumina Inc.). In summary, poly-A containing mRNA molecules were isolated using poly-T oligo-attached magnetic beads; then, a random chemical fragmentation of the mRNA was followed by cDNA synthesis, ligation of Illumina's sequencing universal adaptors and PCR amplification of ligated fragments. Libraries containing inserts between 80 and $120 \mathrm{bp}$ (libraries sizes ranging from 200 to $250 \mathrm{bp}$ ) were quantified by Q-PCR, clustered and amplified on flow-cell lanes and sequenced in a Genome Analyzer II (Illumina Inc.) for 36 or 38 cycles. The sequences were submitted to NCBI Sequence Read Archive (SRA) (http://trace.ncbi.nlm.nih.gov/Traces/sra/ sra.cgi?view=studies) under submission ID: SRA048981.1 and to Gene Expression Omnibus (GEO) database under accession number GSE38664.

Short reads were aligned to the Drosophila melanogaster genome version FlyBase r5.22 with Bowtie [63] allowing using default options, but selecting only the best alignment (--best). Reads were annotated with the R/Bioconductor Genominator package and 
differential expression measured with the DESeq package [19]. Aligned reads were also normalized by RPKMs [20].

\section{Gene Ontology and other bioinformatics analysis}

Preliminary non-biased gene ontology (GO) analysis was carried out using VLAD online tool [64]. Then, functional groups lists of genes were manually elaborated based on the literature and the following databases: VLAD [64], AMIGO [65], GATHER [66], GOTM [67] and FlyBase [68]. Homozygous sall mutant misregulated genes found in our analysis (at stage 16, 17 and at the transition between both stages), were then classified in our "Functional Groups reference lists". The following functional groups were constructed from specific literature sources: Tissue regeneration [21]; Salt stress (genes overexpressed 4hs after salt treatment, [24]); Mitochondrial diseases [40]; Lifespan/Aging [59,60,69-71]. Genes belonging to functional groups different from selected "Reference Groups" were categorized under the generic term "Other". Genes with no function experimentally demonstrated or suggested yet, were classified as "Unknown function". We then calculated and graphically represented the percentage of sall mutant misregulated genes at stage 16 (WT16 vs Ho16; He16 vsHo16), 17 (WT17 vs Ho17; He17 vs Ho17) and at the transition between both stages (Ho16 vs Ho17; WT16 vs WT17) belonging to each "Functional Group reference list". Finally, we performed Gene Ontology (GO) analysis using the FatiGO software of the BABELOMICS package [72]. Additional file 14: Table S14 shows the genes in each Functional Group category used in this study.

For the analysis of Sall binding sites we used the MatScan software [73], demanding at most 1 substitution from the published consensus [11] and using the BDGP R5/dm3 genome assembly as reference.

We compared our sequencing data with those from Graveley et al. [17] in two different ways: 1) We calculated Pearson correlation coefficients between the modENCODE RNA-seq data from 12 to $14 \mathrm{~h}$ embryos (SRX015647) and our WT16 data and between the modENCODE RNA-seq data from 18 to $20 \mathrm{~h}$ embryos (SRX015650) and WT17; and 2) we determined the number of shared genes in the WT 16/17 and 12-14 h/ 18-20 h transitions, both for up and down-regulated. For both analyses we carried out first a mediannormalization of the data in order to transform the expression values into a comparable scale. In the second analysis we consider a gene to be differentially expressed if it is at least 4-fold over- or under-expressed.

For the comparison of our data with that from the silencing of salm in the muscle, we compared the homozygous sall mutant misregulated genes found in our experiment with the top 500 genes of the microarrays of salm downregulated pupal muscle [46].

\section{Additional files}

Additional file 1: Table S1. Sequencing parameters of the biological replicas of the studied genotypes. Genotype symbols: Wild type (WT), Heterozygous (He), Homozygous (Ho). Letters a, b and c symbolize different experiments for each genotype. Technical replicates were grouped (ie: WT16ab).

Additional file 2: Table S2. Differential expression analysis. baseMean (mean expression level, at the base scale, as a joint estimate from both conditions); baseMeanA (mean expression level, at the base scale, as an estimate for condition A); baseMeanB (mean expression level, at the base scale, as an estimate for condition B); foldChange (fold change from the first to the second condition); log2FoldChange (logarithm to basis 2 of the fold change); pvalue (significance of the fold change); padjusted ( $p$ values adjusted for multiple testing with the Benjamini-Hochberg procedure, which controls false discovery rate); resVarA (ratio of the single gene estimates for the base variance to the fitted value in condition A); resVarB (ratio of the single gene estimates for the base variance to the fitted value in condition B).

Additional file 3: Table S3. RPKM normalization of gene counts. Genotype symbols: Wild type (WT), Heterozygous (He), Homozygous (Ho). Letters $a, b$ and $c$ symbolize different experiments for each genotype. Technical replicates for a same genotype sample were grouped (ie: WT16ab).

Additional file 4: Table S4. Number of genes significantly misregulated in homozygous or heterozygous sall mutant embryos in comparison to WT. Genotype symbols: Wild type (WT), Heterozygous (He), Homozygous (Ho). Downregulated (D) or upregulated (U) genes in the second genotype with respect to the first one; $E$, no significant changes in expression between the two genotypes.

Additional file 5: Figure S1. Dosage effect of sall genes. (A) mRNASeq expression plot for arm, Fas-3, Nrg, Fas2, CadN and N genes in heterozygous (He) and homozygous (Ho) sall mutant embryos at stage 16 showed consistent results with the differences in protein levels observed by Cantera et al. (Cantera et al. 2002). Notice that heterozygous sall mutant embryos have higher transcript levels than homozygous for all these adhesion and cytoskeleton genes, suggesting a dosage effect of Sall. (B) mRNA-Seq analysis of the transcriptome of WT16, He16 and Ho16 embryos, showed that five genes are differentially expressed $(p<0.01)$ between all the genotypes compared at stage 16 and have intermediate levels of expression in He16. (C) At stage 17, instead, four of the genes that are differentially expressed $(p<0.01)$ between all the genotypes compared at this stage had intermediate levels of expression in He17.

Additional file 6: Figure S2. Reads mapped along IM3 and dro5 genes. (A, B) 36 or 38 long reads represented by grey lines map on dro5 (A) or IM3 (B) genes. Gene, mRNA and coding sequence (CDS) are represented on the upper part of each figure. On the left, the different biological replicates of each genotype analyzed are indicated.

Additional file 7: Figure S3. Putative Sall binding sites in regulated genes. Graphical representation of $\operatorname{dro5}(\mathrm{A})$ and IM3 (B) genes and the putative Sall binding sites (pink box in $A$, green and blue boxes in $B$ ) in the genomic region. Conservation of these sequences in various Drosophila species is depicted below the graphs.

Additional file 8: Table S5. Misregulated genes in homozygous sall mutant embryos. Genotype symbols: Wild type (WT), Heterozygous (He), Homozygous (Ho). Downregulated (D) or upregulated (U) genes in the second genotype with respect to the first one; E, no significant changes in expression between the two genotypes. Genes previously associated with neurodegeneration/neuroprotection are indicated in RED.

Additional file 9: Unbiased Gene Ontology analysis of the genes significantly misregulated in homozygous sall mutant embryos using the VLAD online tool. Genes misregulated in homozygous sall mutant embryos at stage 16,17 and at the transition between both 
stages (genotype comparisons: WT16 vs Ho16; WT17 vs Ho17; Ho16 vs Ho17).

Additional file 10: Table S6. Functional classification of genes misregulated in homozygous sall mutant embryos with respect to wild type embryos at stage 16. Genotype comparison WT16 vs Ho16. Wild type (WT), Homozygous (Ho).Table S7. Functional classification of genes misregulated in homozygous sall mutant embryos with respect to heterozygous embryos at stage 16 . Genotype comparison Ho16 vs He16. Homozygous (Ho), Heterozygous (He). Table S8. Functional classification of genes misregulated in homozygous sall mutant embryos with respect to wild type embryos at stage 17. Genotype comparison WT17 vs Ho17. Wild type (WT), Homozygous (Ho). Table S9. Functional classification of genes misregulated in homozygous sall mutant embryos with respect to heterozygous embryos at stage 17. Genotype comparison Ho17 vs He17. Homozygous (Ho), Heterozygous (He). Table S10. Functional classification of genes misregulated in homozygous sall mutant embryos at the transition from stage 16 to 17 . Genotype comparison Ho16 vs Ho17. Homozygous (Ho). Table S11. Functional classification of genes misregulated in wild type embryos at the transition from stage 16 to 17. Genotype comparison WT16 vs WT17. Wild type (WT).

Additional file 11: Figure S4. Functional classification of misregulated genes. (A-C) Graphic representation of the main functional groups enriched in misregulated genes in the indicated genotypes, expressed as percentage of genes in each group. In red are marked the groups significantly overrepresented with respect to the total Drosophila genome with $p<0.01$ and in green with $p<0.05$ (A) Classification of the genes misregulated in Ho16 compared with He16. (B) Classification of the genes misregulated in Ho17 compared to He17. (C) Classification of the genes misregulated in WT embryos at the transition from stage 16 to 17 .

Additional file 12: Table S12. Genes regulated by Sall both in embryonic stages and in and pupal muscle.

Additional file 13: Table S13. Oligonucleotides used for PCR analysis.

Additional file 14: Table S14. Functional Groups reference list. ${ }^{*} G O$ categories constructed from specific literature sources.

\section{Abbreviations}

CNS: Central Nervous System; GFP: Green Fluorescent Protein; GO: Gene Ontology; He: Heterozygotes; Ho: Homozygotes; mRNAseq: mRNA sequencing; Q-PCR: Quantitative Polimerase Chain Reaction; RPKM: Reads Per Kilobase of transcript per Million mapped reads; Salm: Spalt major; Salr: Spalt related; WT: Wild type.

\section{Competing interests}

The authors declare that they have no competing interests.

\section{Authors' contributions}

MJF designed and performed all the molecular genetic experiments, participated in the interpretation of the results and in the writing of the manuscript. NR participated in the bioinformatic analyses and in the interpretation of the results. CP provided the fly stocks. MH participated in the bioinformatic analyses. AA coordinated and performed the mRNA sequencing and participated in the interpretation of the results and the bioinformatic analyses. RB and RC conceived, designed and coordinated the study, participated in the interpretation of the results and in the writing of the manuscript. All authors read and approved the final manuscript.

\section{Acknowledgements}

We thank E. Blanco from the Bioinformatics Platform of the Genshape Consolider Program and J. L. Lavín from the Genome Analysis Platform at CIC bioGUNE for their help with the data analysis and J. D. Sutherland for critical reading of the manuscript. RB thanks the Spanish MICINN (grants BFU2008-01884, BFU2011-25986 and the Consolider Program CSD2007-00825120), the Departments of Education and Industry of the Basque Government (PI2009-16, PI2012-42 and Etortek Research Programs 2008/2009 and 2009/2011) and the Bizkaia County. RC received funds from the Sistema
Nacional de Investigadores (Uruguay) and the Carl Trygger Foundation (Sweden).

\section{Author details}

${ }^{1}$ Developmental Neurobiology, IIBCE, Montevideo, Uruguay. ${ }^{2}$ Genome Analysis Platform, CIC bioGUNE \& CIBERehd, Derio, Spain. ${ }^{3}$ Current affiliation: AZTI Tecnalia, Marine Research Division, Sukarrieta, Spain. ${ }^{4}$ Functional Genomics, ClC bioGUNE, Derio, Spain. ${ }^{5}$ Genetics Department, Granada University, Granada, Spain. ${ }^{6}$ Zoology Department, Stockholm University, Stockholm, Sweden.

Received: 27 March 2012 Accepted: 11 September 2012

Published: 15 September 2012

\section{References}

1. Hirth F: Drosophila melanogaster in the study of human neurodegeneration. CNS Neurol Disord 2010, 9:504-523.

2. Lessing $D$, Bonini NM: Maintaining the brain: insight into human neurodegeneration from Drosophila melanogaster mutants. Nat Rev Genet 2009, 10:359-370.

3. Lu B: Recent advances in using Drosophila to model neurodegenerative diseases. Apoptosis 2009, 14:1008-1020.

4. Elliott DA, Brand AH: The GAL4 system: a versatile system for the expression of genes. Meth Mol Biol 2008, 420:79-95.

5. Kohlhase J, Wischermann A, Reichenbach H, Froster U, Engel W: Mutations in SALL1 putative transcription factor gene cause Townes-Brocks syndrome. Nat Genet 1998, 18:81-83.

6. Al-Baradie R, Yamada K, St Hilaire C, Chan WM, Andrews C, Mclntosh N, Nakano M, Martonyi EJ, Raymond WR, Okumura S, Okihiro MM, Engle EC Duane radial ray syndrome (Okihiro syndrome) maps to $20 \mathrm{q} 13$ and results from mutations in SALL4, a new member of the SAL family. Am J Med Genet 2002, 71:1195-1199.

7. Kohlhase J, Heinrich M, Schubert L, Liebers M, Kispert A, Laccone F, Turnpenny P, Winter RM, Reardon W: Okihiro syndrome is caused by SALL4 mutations. Hum Mol Genet 2002, 11:2979-2987.

8. Cameron TH, Lachiewicz AM, Aylsworth AS: Townes-Brocks syndrome in two mentally retarded youngsters. Am J Med Genet 1991, 41:1-4.

9. Ishikiriyama S, Kudoh F, Shimojo N, Iwai J, Inoue T: Townes-Brocks syndrome associated with mental retardation. Am J Med Genet 1996, 61:191-192.

10. de Celis JF, Barrio R: Regulation and function of Spalt proteins during animal development. Int J Dev Biol 2009, 53:1385-1398.

11. Barrio R, Shea MJ, Carulli J, Lipkow K, Gaul U, Frommer G, Schuh R, Jäckle H, Kafatos FC: The spalt-related gene of Drosophila melanogaster is a member of an ancient gene family, defined by the adjacent, regionspecific homeotic gene spalt. Dev Genes Evol 1996, 206:315-325.

12. Kuhnlein RP, Schuh R: Dual function of the region-especific homeotic gene spalt during Drosophila tracheal system development. Development 1996, 122:2215-2223.

13. Cantera R, Lüer K, Rusten TE, Barrio R, Kafatos FC, Technau GM: Mutations in spalt cause a severe but reversible neurodegenerative phenotype in the embryonic central nervous system of Drosophila melanogaster. Development 2002, 129:5577-5586.

14. Rusten TE, Cantera R, Urban J, Technau G, Kafatos FC, Barrio R: Spalt modifies EGFR-mediated induction of chordotonal precursors in the embryonic PNS of Drosophila promoting the development of oenocytes. Development 2001, 128:711-722.

15. Arbeitman MN, Furlong EE, Imam F, Johnson E, Null BH, Baker BS, Krasnow MA, Scott MP, Davis RW, White KP: Gene expression during the life cycle of Drosophila melanogaster. Science 2002, 297:2270-2275.

16. Hooper SD, Boué S, Krause R, Jensen LJ, Mason CE, Ghanim M, White KP, Furlong EE, Bork P: Identification of tightly regulated groups of genes during Drosophila melanogaster embryogenesis. Mol Syst Biol 2007, 3:72.

17. Graveley BR, Brooks AN, Carlson JW, Duff MO, Landolin JM, Yang L, Artieri CG, van Baren MJ, Boley N, Booth BW, Brown JB, Cherbas L, Davis CA, Dobin A, Li R, Lin W, Malone JH, Mattiuzzo NR, Miller D, Sturgill D, Tuch BB, Zaleski C, Zhang D, Blanchette M, Dudoit S, Eads B, Green RE, Hammonds A, Jiang $L$, Kapranov P, et al: The developmental transcriptome of Drosophila melanogaster. Nature 2011, 471:473-479.

18. Barrio R, de Celis JF, Bolshakov S, Kafatos FC: Identification of regulatory regions driving the expression of the Drosophila spalt complex at different developmental stages. Dev Biol 1999, 215:33-47. 
19. Anders S, Huber W: Differential expression analysis for sequence count data. Genome Biol 2010, 11:R106.

20. Mortazavi A, Williams BA, McCue K, Schaeffer L, Wold B: Mapping and quantifying mammalian transcriptomes by RNA-Seq. Nat Methods 2008, 5:621-628.

21. Blanco E, Ruiz-Romero M, Beltran S, Bosch M, Punset A, Serras F, Corominas $\mathrm{M}$ : Gene expression following induction of regeneration in Drosophila wing imaginal discs. Expression profile of regenerating wing discs. BMC Dev Biol 2010, 10:94.

22. Calleja M, Pena P, Ugalde C, Ferreiro C, Marco R, Garesse R: Mitochondrial DNA remains intact during Drosophila aging, but the levels of mitochondrial transcripts are significantly reduced. J Biol Chem 1993, 268:18891-18897.

23. Seong KM, Kim CS, Seo SW, Jeon HY, Lee BS, Nam SY, Yang KH, Kim JY, Kim CS, Min KJ, Jin YW: Genome-wide analysis of low-dose irradiated male Drosophila melanogaster with extended longevity. Biogerontology 2011 12:93-107.

24. Stergiopoulos K, Cabrero P, Davies SA, Dow JA: Salty dog, and SCL5 symporter, modulates Drosophila response to salt stress. Physiol Genomics 2009, 37:1-11.

25. Scherzer CR, Jensen RV, Gullans SR, Feany MB: Gene expression changes presage neurodegeneration in a Drosophila model of Parkinson's disease. Hum Mol Genet 2003, 12:2457-2466.

26. Zinke I, Schütz CS, Katzenberger JD, Bauer M, Pankratz MJ: Nutrient contro of gene expression in Drosophila: microarray analysis of starvation and sugar-dependent response. EMBO J 2002, 21:6162-6173.

27. Li HM, Sun L, Mittapalli O, Muir WM, Xie J, Wu J, Schemerhorn BJ, Sun W, Pittendrigh BR, Murdock LL: Transcriptional signatures in response to wheat germ agglutinin and starvation in Drosophila melanogaster larval midgut. Insect Mol Biol 2009, 18:21-31.

28. Borozdin W, Boehm D, Leipoldt M, Wilhelm C, Reardon W, Clayton-Smith J, Becker K, Mühlendyck H, Winter R, Giray O, Silan F, Kohlhase J: SALL4 deletions are a common cause of Okihiro and acro-renal-ocular syndromes and confirm haploinsufficiency as the pathogenic mechanism. J Med Genet 2004, 41:e113.

29. de Celis JF, Barrio R: Function of the spalt/spalt-related gene complex in positioning the veins in the Drosophila wing. Mech Dev 2000, 91:31-41.

30. Papatsenko I, Levine M, Papatsenko D: Temporal waves of coherent gene expression during Drosophila embryogenesis. Bioinformatics 2010, 26:2731-2736.

31. Sánchez J, Talamillo A, González M, Sánchez-Pulido L, Jiménez S, Pirone L, Sutherland JD, Barrio R: Drosophila Sal and Salr are transcriptional repressors. Biochem J 2011, 438:437-445.

32. Netzer C, Rieger L, Brero A, Zhang CD, Hinzke M, Kohlhase J, Bohlander SK SALL1, the gene mutated in Townes-Brocks syndrome, encodes a transcriptional repressor which interacts with TRF1/PIN2 and localizes to pericentromeric heterochromatin. Hum Mol Genet 2001, 10:3017-3024.

33. Kiefer SM, MCDill BW, Yang J, Rauchman M: Murine Sall1 represses transcription by recruiting a histone deacetylase complex. J Biol Chem 2002, 277:14869-14876.

34. Greene JC, Whitworth AJ, Andrews LA, Parker TJ, Pallanck LJ: Genetic and genomic studies of Drosophila parkin mutants implicate oxidative stress and innate immune responses in pathogenesis. Hum Mol Genet 2005, 14:799-811.

35. Nelson B, Nishimura $S$, Kanuka H, Kuranaga E, Inoue M, Hori G, Nakahara H, Miura M: Isolation of gene sets affected specifically by polyglutamine expression: implication of the TOR signaling pathway in neurodegeneration. Cell Death Differ 2005, 12:1115-1123.

36. Häcker U, Lin X, Perrimon N: The Drosophila sugarless gene modulates Wingless signaling and encodes an enzyme involved in polysaccharide biosynthesis. Development 1997, 124:3565-3573.

37. Monnier V, Girardot F, Audin W, Tricoire H: Control of oxidative stress resistance by IP3 kinase in Drosophila melanogaster. Free Radic Biol Med 2002, 33:1250-1259.

38. Rival T, Page RM, Chandraratna DS, Sendall TJ, Ryder E, Liu B, Lewis $H$, Rosahl T, Hider R, Camargo LM, Shearman MS, Crowther DC, Lomas DA: Fenton chemistry and oxidative stress mediate the toxicity of the betaamyloid peptide in a Drosophila model of Alzheimer's disease. Eur J Neurosci 2009, 29:1335-1347.

39. Gruenewald C, Botella JA, Bayersdorfer F, Navarro JA, Schneuwly S: Hyperoxia-induced neurodegeneration as a tool to identify neuroprotective genes in Drosophila melanogaster. Free Radic Biol Med 2009, 46:1668-1676

40. Fernández-Ayala DJ, Chen S, Kemppainen E, O'Dell KM, Jacobs HT: Gene expression in a Drosophila model of mitochondrial disease. PLoS One 2010, 5:e8549

41. Jenner P: Oxidative stress in Parkison's disease. Ann Neurol 2003 53:S26-\$38.

42. Yun SW, Gerlach M, Riederer P, Klein MA: Oxidative stress in the brain at early preclinical stages of mouse scrapie. Exp Neurol 2006, 201:90-98

43. Sau D, De Biasi S, Vitellaro-Zuccarello L, Riso P, Guarnieri S, Porrini M, Simeoni S, Crippa V, Onesto E, Palazzolo I, Rusmini P, Bolzoni E, Bendotti C, Polleti A: Mutation of SOD1 in ALS: gain of a loss of function. Hum Mol Genet 2007, 16:1604-1618.

44. Trushina E, McMurray CT: Oxidative stress and mitochondrial dysfunction in neurodegenerative diseases. Neuroscience 2007, 145:1233-1248.

45. Zhu X, Lee HG, Perry G, Smith MA: Alzheimer disease, the two-hit hypothesis: an update. Biochim Biophys Acta 2007, 1772:494-502.

46. Schönbauer C, Distler J, Jährling N, Radolf M, Dodt HU, Frasch M, Schnorrer F: Spalt mediates an evolutionarily conserved switch to fibrillar muscle fate in insects. Nature 2011, 479:406-409.

47. Aigaki T, Seong KH, Matsuo T: Longevity determination genes in Drosophila melanogaster. Mech Ageing Dev 2002, 123:1531-1541.

48. Mastushita-Sakai T, White-Grindley E, Samuelson J, Seidel C, Si K: Drosophila Orb2 targets genes involved in neuronal growth, synapse formation, and protein turnover. Proc Natl Acad Sci USA 2010, 107:11987-11992.

49. Bonini NM: Chaperoning brain degeneration. Proc Natl Acad Sci USA 2002, 99:16407-16411.

50. Fernandez-Funez P, Casas-Tinto S, Cepeda-Nieto A, Soto C, Rincon-Limas D: Hsp70 protects Drosophila brain neurons against Prion-dependent neurodegeneration. A Dros Res Conf 2007, 48:77

51. Inlow JK, Restifo LL: Molecular and comparative genetics of mental retardation. Genetics 2004, 166:835-881.

52. Govind S: Control of development and immunity by rel transcription factors in Drosophila. Oncogene 1999, 18:6875-6887.

53. Kaltschmidt B, Widera D, Kaltschmidt C: Signaling via NF-kappaB in the nervous system. Biochim Biophys Acta 2005, 1745:287-299.

54. Xu Z, Jung C, Higgins C, Levine J, Kong J: Mitochondrial degeneration in amyotrophic lateral sclerosis. J Bioenerg Biomembr 2004, 36:395-399.

55. Harbison ST, Chang S, Kamdar KP, Mackay TF: Quantitative genomics of starvation stress resistance in Drosophila. Genome Biol 2005, 6:R36.

56. Fujikawa K, Takahashi A, Nishimura A, Itoh M, Takano-Shimizu T, Ozaki M: Characteristics of genes up-regulated and down-regulated after $24 \mathrm{~h}$ starvation in the head of Drosophila. Gene 2009, 446:11-17.

57. Palanker L, Tennessen JM, Lam G, Thummel CS: Drosophila HNF4 regulates lipid mobilization and beta-oxidation. Cell Metab 2009, 9:228-239.

58. West AP Jr, Llamas LL, Snow PM, Benzer S, Bjorkman PJ: Crystal structure of the ectodomain of Methuselah, a Drosophila G protein-coupled receptor associated with extended lifespan. Proc Natl Acad Sci USA 2001, 98:3744-3749.

59. Curtis C, Landis GN, Folk D, Wehr NB, Hoe N, Waskar M, Abdueva D, Skvortsov D, Ford D, Luu A, Badrinath A, Levine RL, Bradley TJ, Tavaré S, Tower J: Transcriptional profiling of MnSOD-mediated lifespan extension in Drosophila reveals a species-general network of aging and metabolic genes. Genome Biol 2007, 8:R262.

60. Lai CQ, Parnell LD, Lyman RF, Ordovas JM, Mackay TF: Candidate genes affecting Drosophila life span identified by integrating microarray gene expression analysis and QTL mapping. Mech Ageing Dev 2007 128:237-249.

61. Boyd O, Weng P, Sun X, Alberico T, Laslo M, Obenland DM, Kern B, Zou S: Nectarine promotes longevity in Drosophila melanogaster. Free Radic Biol Med 2011, 50:1669-1678.

62. National Center for Biotechnology Information. http://www.ncbi.nlm.nih.gov/ tools/primer-blast].

63. Langmead B, Trapnell C, Pop M, Salzberg SL: Ultrafast and memoryefficient alignment of short DNA sequences to the human genome. Genome Biol 2009, 10:R25.

64. Visual Annotation Display. http://informatics.jax.org/ jer/vlad.

65. The Gene Ontology. http://amigo.geneontology.org.

66. Gene Annotation Tool to Help Explain Relationships. http://gather.genome. duke.edu. 
67. GOTree Machine. http://genereg.ornl.gov/gotm.

68. FlyBase: A Database of Drosophila Genes \& Genomes. http://flybase.org

69. Camporeale G, Giordano E, Rendina R, Zempleni J, Eissenberg JC:

Drosophila melanogaster holocarboxylase synthetase is a chromosomal protein required for normal histone biotinylation, gene transcription patterns, lifespan, and heat tolerance. J Nutr 2006, 136:2735-2742.

70. Lee KS, lijima-Ando K, lijima K, Lee WJ, Lee JH, Yu K, Lee DS: JNK/FOXOmediated neuronal expression of fly homologue of peroxiredoxin II reduces oxidative stress and extends life span. J Biol Chem 2009, 284:29454-29461.

71. Kim HJ, Morrow G, Westwood JT, Michaud S, Tanguay RM: Gene expression profiling implicates OXPHOS complexes in lifespan extension of flies over-expressing a small mitochondrial chaperone, Hsp22. Exp Gerontol 2010, 45:611-620.

72. Al-Shahrour F, Minguez P, Tárraga J, Montaner D, Alloza E, Vaquerizas JM, Conde L, Blaschke C, Vera J, Dopazo J: BABELOMICS: a systems biology perspective in the functional annotation of genome-scale experiments. Nucleic Acid Res 2006, 34:W472-W476.

73. Blanco E, Messeguer X, Smith TF, Guigó R: Transcription factor map alignment of promoter regions. PLoS Comput Biol 2006, 2:e49.

doi:10.1186/1471-2164-13-483

Cite this article as: Ferreiro et al.: Whole transcriptome analysis of a reversible neurodegenerative process in Drosophila reveals potential neuroprotective genes. BMC Genomics 2012 13:483.

\section{Submit your next manuscript to BioMed Central and take full advantage of:}

- Convenient online submission

- Thorough peer review

- No space constraints or color figure charges

- Immediate publication on acceptance

- Inclusion in PubMed, CAS, Scopus and Google Scholar

- Research which is freely available for redistribution 